\title{
lodine-related attenuation in contrast- enhanced dual-energy computed tomography in small-sized solid-type lung cancers is associated with the postoperative prognosis
}

\author{
Shingo Iwano ${ }^{1 *} \mathbb{D}$, Shinichiro Kamiya ${ }^{1}$, Rintaro Ito ${ }^{1}$, Shota Nakamura ${ }^{2}$ and Shinji Naganawa ${ }^{1}$
}

\begin{abstract}
Background: To investigate the correlation between iodine-related attenuation in contrast-enhanced dual-energy computed tomography (DE-CT) and the postoperative prognosis of surgically resected solid-type small-sized lung cancers.

Methods: We retrospectively reviewed the DE-CT findings and postoperative course of solid-type lung cancers $\leq 3$ $\mathrm{cm}$ in diameter. After injection of iodinated contrast media, arterial phases were scanned using $140-\mathrm{kV} p$ and $80-\mathrm{kVp}$ tube voltages. Three-dimensional iodine-related attenuation (3D-IRA) of primary tumors at the arterial phase was computed using the "lung nodule" application software. The corrected 3D-IRA normalized to the patient's body weight and contrast medium concentration was then calculated.

Results: A total of 120 resected solid-type lung cancers $\leq 3 \mathrm{~cm}$ in diameter were selected for analysis (82 males and 38 females; mean age, 67 years). During the observation period (median, 47 months), 32 patients showed postoperative recurrence. Recurrent tumors had significantly lower 3D-IRA and corrected 3D-IRA at early phase compared to non-recurrent tumors ( $p=0.046$ and $p=0.027$, respectively). The area under the receiver operating characteristic curve for postoperative recurrence was 0.624 for the corrected 3D-IRA at early phase $(p=0.025)$, and the cutoff value was 5.88. Kaplan-Meier curves for disease-free survival indicated that patients showing tumors with 3D-IRA $>5.88$ had a significantly better prognosis than those with tumors showing 3D-IRA $<5.88(p=0.017)$.

Conclusions: The 3D-IRA of small-sized solid-type lung cancers on contrast-enhanced DE-CT was significantly associated with postoperative prognosis, and low 3D-IRA tumors showed a higher TNM stage and a significantly poorer prognosis.
\end{abstract}

Keywords: Contrast enhancement, Dual-energy CT, Functional imaging, lodine-related attenuation, Lung cancer

\footnotetext{
* Correspondence: iwano45@med.nagoya-u.ac.jp

'Department of Radiology, Nagoya University Graduate School of Medicine,

65 Tsurumai-cho, Showa-ku, Nagoya 466-8550, Japan

Full list of author information is available at the end of the article
}

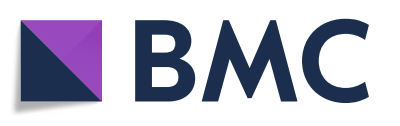

(- The Author(s). 2021 Open Access This article is licensed under a Creative Commons Attribution 4.0 International License, which permits use, sharing, adaptation, distribution and reproduction in any medium or format, as long as you give appropriate credit to the original author(s) and the source, provide a link to the Creative Commons licence, and indicate if changes were made. The images or other third party material in this article are included in the article's Creative Commons licence, unless indicated otherwise in a credit line to the material. If material is not included in the article's Creative Commons licence and your intended use is not permitted by statutory regulation or exceeds the permitted use, you will need to obtain permission directly from the copyright holder. To view a copy of this licence, visit http://creativecommons.org/licenses/by/4.0/. The Creative Commons Public Domain Dedication waiver (http://creativecommons.org/publicdomain/zero/1.0/) applies to the data made available in this article, unless otherwise stated in a credit line to the data. 


\section{Introduction}

The prognosis of primary lung cancer is affected by many clinical, imaging, and histopathological findings. In general, small-sized lung cancers with a diameter equal to or less than $3 \mathrm{~cm}$ have a good postoperative prognosis $[1,2]$. In addition, the ratio of solid component and ground-glass opacity within a nodule on thin-section CT images significantly contributes to its prognosis [2-5]. Therefore, the solid size in a nodule has been adopted as a $\mathrm{T}$ factor in the recent TNM staging. However, several recent studies have shown that solid nodules have a worse prognosis than part-solid nodules with groundglass opacity, even if the solid sizes of tumors are the same [6-10]. For resectable lung cancers, preoperative diagnostic imaging is essential for determining the best therapeutic strategy, including the type of surgical procedure. Therefore, there is a need for imaging techniques that can predict the postoperative prognosis of small-sized solid lung cancers.

A dual-energy CT (DE-CT) technique using two types of tube voltages can enable quantification of the iodinerelated attenuation (IRA) of iodinated contrast material in the tumor after intravenous injection without the need for an additional non-contrast CT scan [11-14], thereby reducing radiation exposure during a CT examination. Several research findings indicate that iodine uptake in the arterial phase on contrast CT of primary lung cancer may be associated with some aspects of tumor histopathology such as angiogenesis, differentiation grade, hypoxic cells, and tumor invasiveness [12, 15-18]. Moreover, a few studies indicated that iodine uptake of lung cancer correlated with tumor recurrence after radiotherapy and chemoradiotherapy [19, 20]. Therefore, we speculated that the three-dimensional iodine-related attenuation (3D-IRA) of solid nodules might be associated with the postoperative recurrence of small-sized lung cancers. If that is true, a dual-energy technique would enable preoperative prediction of the prognosis. To date, no study has investigated the correlation between contrast-enhanced dual-energy CT findings and postoperative recurrence in lung cancer.

In this study, we reviewed preoperative data from contrast CT with dual-energy scanning as well as postoperative pathological TNM staging and disease-free survival of resected of lung cancers $\leq 3 \mathrm{~cm}$, and validated the hypothesis that the 3D-IRA of small-sized solid lung cancers could be a predictive factor for the prognosis.

\section{Methods and materials}

\section{Patient selection}

We reviewed the medical records, postoperative pathological records, and preoperative DE-CT images of patients who underwent surgical lung resection for primary lung cancer (adenocarcinoma, squamous cell carcinomas, adenosquamous carcinomas, large cell neuroendocrine carcinomas, small cell carcinoma, pleomorphic carcinoma, and lymphoepithelioma-like carcinoma) at our hospital between April 2014 and March 2016 (Fig. 1). We limited tumors $\leq 3 \mathrm{~cm}$ in diameter that had been definitively diagnosed by pathological examination after surgical resection. Pure and part-solid ground-glass nodules were excluded. Patients who received neoadjuvant chemoradiotherapy were also excluded. We recorded patient characteristics (gender, age, and body weight) from medical records, and tumor characteristics (pathological tumor size [pSize], histological type, differentiation grade, pathological TNM staging [pStage]) from pathological records. Herein, the seventh edition of union for international cancer control (UICC) TNM staging was used. Cases that were recurrence-free and had a postoperative follow-up period of less than 1 year were excluded. The diagnosis of recurrence was made by thoracic surgeons through routine check-ups by tumor markers, chest X-ray, chest and abdomen CT, and head MRI. PET/CT and biopsies were added to determine the eventual diagnosis in cases of suspected recurrence.

\section{CT scan protocol}

All CT scans were performed with a dual-source CT scanner (SOMATOM Definition Flash; Siemens Healthcare, Tokyo, Japan) in the craniocaudal direction with inspiratory apnea. For vessel enhancement, $96 \mathrm{~mL}$ of non-ionic contrast medium (Proscope 300, $300 \mathrm{mgI} / \mathrm{mL}$ iopromide, Alfresa Pharma, Osaka, Japan; Optiray 320, $320 \mathrm{mgI} / \mathrm{mL}$ ioversol, Tyco Healthcare, Tokyo, Japan; or iopamiron 370, $370 \mathrm{mgI} / \mathrm{mL}$ iopamidol, Bayer Healthcare, Tokyo, Japan) was used with flow rates of $4.0 \mathrm{~mL} /$ sec. Iopromide was used for patients with body weights $<40 \mathrm{~kg}$, ioversol was used for patients with body weights $40-59 \mathrm{~kg}$, and iopamidol was used for patients with body weights $\geq 60 \mathrm{~kg}$. Injections were immediately followed by a saline chaser bolus of $20 \mathrm{~mL}$ at the same flow rate using a dual-barrel power injector (Dual Shot GX7; Nemoto Kyorindo, Tokyo, Japan).

At our institution, dual-phase contrast-enhanced CTs were performed routinely for preoperative evaluation of lung cancer. The early-phase thoracic CT images were scanned for preoperative evaluation of pulmonary vessels by $3 \mathrm{D}$ pulmonary angiography. The scan delay was evaluated by an automatic bolus tracking system with a circular ROI localized on the thoracic aorta. Scanning started automatically as the attenuation of the ROI increased by $70 \mathrm{HU}$ from baseline (about 20-25 s after the start of injection). A dual-energy scan system (tube A at a peak voltage of $140 \mathrm{kVp}$ and tube $\mathrm{B}$ at a peak voltage of $80 \mathrm{kVp}$ ) was used for both scans with collimation of $64 \times 0.6 \mathrm{~mm}$ and gantry rotation speed of $285 \mathrm{~ms}$. Data were reconstructed with a slice thickness of $1.0 \mathrm{~mm}$ at 


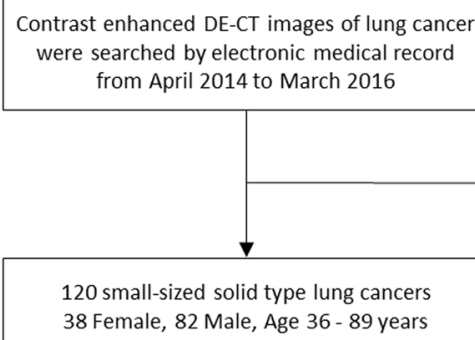

120 small-sized solid type lung cancers

38 Female, 82 Male, Age 36 - 89 years

Fig. 1 Study cohort flowchart. After applying the exclusion criteria, 120 small-sized (pathological tumor size $\leq 3 \mathrm{~cm}$ in diameter) solid-type lung cancers were included in the present analysis

1.0-mm increments using iterative reconstruction techniques (SAFIRE, Siemens Healthcare, Tokyo, Japan). Additionally, delayed-phase thoraco-abdominal CT scans were obtained to evaluate lymph node metastasis and abdominal distant metastasis about 2 min after contrast medium injection.

\section{Analysis of dual-energy CT images}

The 3D-IRA of the primary lesion was measured using the syngo "lung nodules" application accessory software (Siemens Healthcare, Tokyo, Japan). The DE-CT technique can create iodine-enhanced images from DECT raw data sets of $140 \mathrm{kVp}$ and $80 \mathrm{kVp}$, and provide absolute measurements of iodine within the tumors. This application can automatically three-dimensionally segment a pulmonary nodule and calculate the mean IRA of pulmonary nodules with a three-material decomposition algorithm. A chest radiologist with 21 years of experience in reading thoracic CT scans segmented the primary tumor and measured the 3D-IRA in the early and delayed phases.

Additionally, corrected 3D-IRA values to reduce the effect of body weight and iodine concentration of contrast medium were calculated as follows:

$$
\text { Corrected 3D }- \text { IRA }=\frac{\text { raw data of } 3 D-I R A}{\text { Concentration of contrast medium }[\mathrm{mgI}] / \text { Body weight }[\mathrm{kg}]}
$$

\section{Statistical analysis}

First, recurrent and non-recurrent tumors were compared. Gender, histopathological type, and pStage, were compared by chi-squared test. The age, body weight, clinical and pathological tumor size, 3D-IRA, and corrected 3D-IRA were compared by Welch's t-test. Second, correlations between tumor characteristics and pStage IB-III were individually evaluated using univariate logistic regression analysis. Third, areas under the curve of receiver operating characteristic (ROC) analysis for postoperative recurrence were compared with respect to

Table 1 Comparison of patient and tumor characteristics between the recurrence and non-recurrence groups

\begin{tabular}{|c|c|c|c|}
\hline & Non-recurrence & Recurrence & p-value \\
\hline Male / Female (n) & $56 / 32$ & $26 / 6$ & 0.067 \\
\hline Age (years) & $68 \pm 9$ & $67 \pm 10$ & 0.691 \\
\hline Body weight (kg) & $58.8 \pm 10.3$ & $56.4 \pm 9.2$ & 0.217 \\
\hline pSize (mm) & $19.2 \pm 5.7$ & $24.2 \pm 5.7$ & $<0.001$ \\
\hline 3D-IRA at early phase (HU) & $36.7 \pm 19.1$ & $30.0 \pm 14.6$ & 0.046 \\
\hline Corrected 3D-IRA at early phase & $6.18 \pm 3.25$ & $4.94 \pm 2.42$ & 0.027 \\
\hline 3D-IRA at delayed phase (HU) & $45.5 \pm 15.7$ & $40.6 \pm 14.8$ & 0.124 \\
\hline Corrected 3D-IRA at delayed phase & $7.63 \pm 2.51$ & $6.68 \pm 2.37$ & 0.059 \\
\hline Adenocarcinoma / SqCC / Others (n) & $58 / 22 / 8$ & $19 / 9 / 4$ & 0.772 \\
\hline Differentiation grade 1 / 2 / 3 / 4 (n) & $14 / 53 / 17 / 4$ & $4 / 20 / 6 / 2$ & 0.952 \\
\hline cStage IA / IB/ II-III (n) & $60 / 24 / 4$ & $11 / 15 / 6$ & 0.002 \\
\hline pStage IA / IB / II-III (n) & $51 / 24 / 13$ & $7 / 5 / 20$ & $<0.001$ \\
\hline Lobectomy / Segmentectomy /WWR & $78 / 9 / 1$ & $27 / 4 / 1$ & 0.699 \\
\hline Adjuvant therapy none / Available (n) & $53 / 15$ & $13 / 13$ & 0.008 \\
\hline
\end{tabular}


Table 2 Univariate logistic regression analysis of clinical and pathological factors for pathological stage IB-III

\begin{tabular}{lll}
\hline & OR (95\% CI) & p-value \\
\hline Clinical factors & & \\
Male vs. Female & $1.287(0.595-2.780)$ & 0.522 \\
Body weight & $1.007(0.972-1.044)$ & 0.706 \\
Age & $0.988(0.950-1.027)$ & 0.528 \\
Corrected 3D-IRA at early phase & $0.817(0.716-0.933)$ & 0.003 \\
Corrected 3D-IRA at delayed phase & $0.932(0.806-1.078)$ & 0.344 \\
Pathological factors & & \\
Others vs. Adenocarcinoma & $0.838(0.397-1.769)$ & 0.643 \\
pSize & $1.123(1.052-1.200)$ & $<0.001$ \\
Differentiation grade & $1.505(0.899-2.521)$ & 0.120 \\
\hline
\end{tabular}

$3 D$-IRA three-dimensional iodine-related attenuation; $\mathrm{Cl}$ confidence interval; $O R$ odds ratio; $p$ Size pathological tumor size

age, corrected 3D-IRAs, and pSize. Then, Youden's index was used to determine a cutoff level that would indicate recurrence.

Finally, using the cutoff value of 3D-IRA from the ROC analysis, the patients were classified into two groups; disease-free survival (DFS) was assessed using the Kaplan-Meier method; and the survival curves for each group were compared using the log-rank test. DFS was defined as the interval between the surgery and the first disease recurrence, including local recurrence and distant metastasis, or death from any cause.

Analyses were performed using commercial statistical software: Excel 2013 (Microsoft Corp., Redmond, WA) and a statistical add-in (BellCurve version 3.20; Social Survey Research Information Corp, Tokyo, Japan), and SPSS version 23 (IBM Corp., Amonk, NY). A $p$-value $<0.05$ was considered statistically significant.

\section{Results}

A total of 132 resected solid-type lung cancers $\leq 3 \mathrm{~cm}$ in diameter were reviewed. One lesion was excluded because pleural dissemination was noted at the time of surgery. Four recurrence-free lesions were excluded because the follow-up period was shorter than 1 year. Five patients had two and one patient had three synchronous multiple lung cancers. For these patients, the tumor with the highest pathological stage was considered for evaluation, and the remaining seven lesions were excluded.
Finally, 120 consecutive primary lung cancer lesions from 120 patients were selected for analysis $(82$ males and 38 females; mean age, 67 years [range, $36-89$ years]; mean body weight, $58.2 \mathrm{~kg}$ [range, $37-85 \mathrm{~kg}$ ]; mean pSize, $20.5 \mathrm{~mm}$ [range, $8-30 \mathrm{~mm}$ ]). Of these lesions, 77 were adenocarcinomas, 31 were squamous cell carcinomas, four were adenosquamous carcinomas, and two each were large cell neuroendocrine carcinomas, small cell carcinomas, pleomorphic carcinomas, and lymphoepitheliomalike carcinomas. All patients underwent preoperative contrast-enhanced CT and PET/CT staging, and nodal staging was confirmed by endobronchial ultrasound-guided trans-bronchial needle aspiration (EBUS-TBNA) in cases of suspected mediastinal lymph node metastases. In the clinical TNM staging [cStage], 71 tumors were categorized as stage IA, 39 as stage IB, 7 as stage IIA, 1 as IIB, and 2 as IIIA. The surgical procedure was lobectomy in 105 tumors, segmentectomy in 13 tumors and wide wedge resection in 2 tumors. The mean period from CT scan to surgery was $29 \pm 18$ days. In the pStage, 58 tumors were categorized as stage IA, 30 as stage IB, 9 as stage IIA, 7 as IIB, 15 as IIIA, and 1 as IIIB. Postoperative adjuvant chemotherapy was administered to 66 patients and not to 26, but the status for the remaining 26 patients was unknown. The median postoperative observation period was 47 months, with a range of 5-68 months. During the observation period, 32 patients showed postoperative recurrence, and five patients died of diseases other than lung cancer. The median postoperative recurrence interval was 16.5 months, and the range was 5-59 months.

Recurrent tumors had significantly lower 3D-IRA and corrected 3D-IRA at early phase compared to nonrecurrent tumors $(p=0.046$ and $p=0.027$, respectively; Table 1), while no significant difference was found at the delayed phase $(p=0.124$ and $p=0.059$, respectively; Table $1)$. Recurrent tumors showed a significantly larger size than non-recurrent tumors $(p<0.001)$. In addition, recurrent tumors had a significantly higher rate of pathological stage IB or higher $(\mathrm{p}<0.001)$.

The mean \pm standard deviation (SD) corrected 3D-IRA at early phase were $6.68 \pm 3.50$ for stage IA, $4.64 \pm 2.47$ for stage IB, and $5.43 \pm 2.35$ for stage II-III tumors, and 3D-IRA for stage IA tumors was significantly higher than those for stage IB and stage II-III tumors $(p=0.002$

Table 3 Receiver operating characteristic (ROC) analysis for postoperative recurrence

\begin{tabular}{llllll}
\hline Factors & AUC $(\mathbf{9 5} \% \mathbf{C I})$ & p-value & Cutoff value & Sensitivity & Specificity \\
\hline Age & $0.520(0.402-0.639)$ & 0.738 & 72 & 0.531 & 0.648 \\
Corrected 3D-IRA at early phase & $0.624(0.519-0.732)$ & 0.025 & 5.88 & 0.750 & 0.500 \\
Corrected 3D-IRA at delayed phase & $0.598(0.482-0.715)$ & 0.097 & 5.51 & 0.375 & 0.841 \\
pSize & $0.772(0.635-0.848)$ & $<0.001$ & 25 & 0.625 & 0.795 \\
\hline
\end{tabular}

3D-IRA three-dimensional iodine-related attenuation; $\mathrm{Cl}$ confidence interval; $p$ Size pathological tumor size 


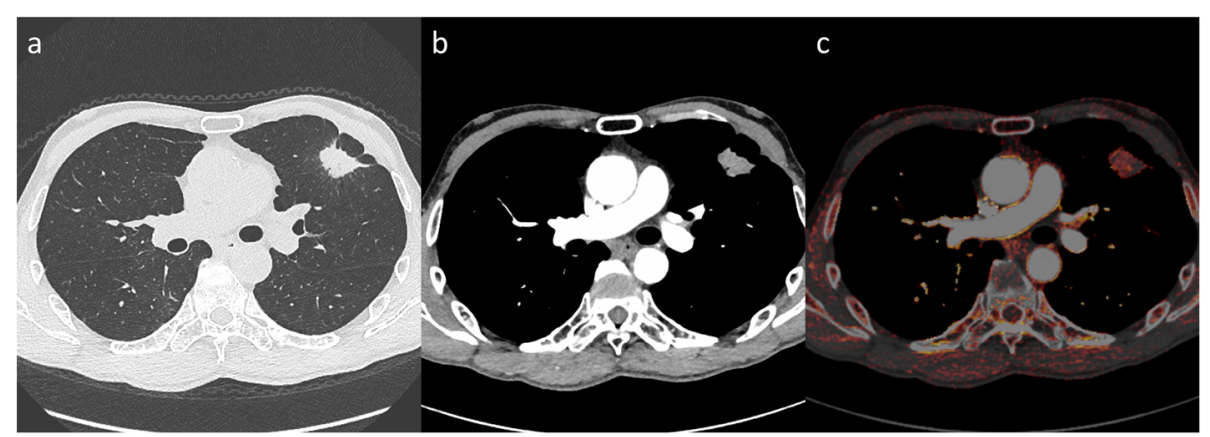

Fig. 2 A 68-year-old male patient. An adenocarcinoma (pT2aN0) is observed in the left upper lobe. a Lung window setting. b Mediastinal window setting at the early phase of contrast-enhanced CT. c lodine-enhanced image in contrast-enhanced CT. The 3D-IRA is 30 HU, and the corrected 3D-IRA is 4.59. The patient developed recurrence of adrenal metastases at 15 months after a left upper lobectomy

and $p=0.034$, respectively). The mean $\pm \mathrm{SD}$ corrected 3D-IRA at early phase were $7.79 \pm 2.81$ for differentiation grade 1 tumors, $5.79 \pm 3.04$ for grade 2 tumors, $4.97 \pm 3.00$ for grade 3 tumors, and $4.22 \pm 2.74$ for grade 4 tumors, and an analysis of variance showed a statistically significant difference among them $(p=0.012)$.

The corrected 3D-IRA at early phase and the tumor size were significantly correlated with stage IB or higher by logistic regression analysis (odds ratio $[\mathrm{OR}]=0.817$, $p=0.003 ; \quad$ and $\mathrm{OR}=1.123, \quad p<0.001 ; \quad$ respectively, Table 2).

The area under the curve (AUC) of ROC analysis for postoperative recurrence was 0.624 for the corrected 3D-IRA at early phase $(p=0.025)$, and the suitable cutoff value was 5.88 with a sensitivity of $75.0 \%$ and specificity of $50.0 \%$ (Table 3 ). When the patients were divided into two groups using this cutoff value, the tumors with corrected $3 D$-IRA $<5.88$ (Fig. 2) had a significantly poorer prognosis than those with 3D-IRA $>5.88$ (Fig. 3) on the Kaplan-Meier survival curve of DFS (mean, 50 months vs. 60 months, $p=0.017$; Fig. $4 \mathrm{a}$ ). Patients with adenocarcinomas, those with squamous cell carcinomas, and those with other subtype tumors showing 3D-IRA $>5.88$ had a better prognosis than those with tumors showing
$3 \mathrm{D}-\mathrm{IRA}<5.88$, although there was no significant difference $(p=0.224, p=0.126$, and $p=0.109$, respectively; Fig. 4b-d,).

\section{Discussion}

It is difficult to preoperatively predict the prognosis of solid-type lung cancers not containing ground-glass opacity. Small-sized lung cancers less than $3 \mathrm{~cm}$, equivalent to T1 in TNM classification, have a good prognosis, although solid-type tumors often recur [6-10]. This study was designed to assess the role of contrast-enhanced DE-CT in predicting the postoperative prognosis of small-sized solid lung cancers resected surgically. We researched the following points: (1) Is the 3D-IRA calculated by DE-CT associated with the TNM staging, even in solid-type tumors $\leq 3 \mathrm{~cm}$ in diameter? (2) Are the 3DIRAs associated with their postoperative DFS? This study revealed that the $3 \mathrm{D}$-IRA at early phase was significantly correlated with pathological TNM staging. The low-3D-IRA tumors tended to show higher TNM stage. In addition, 3D-IRA at early phase was correlated with postoperative recurrence; tumors with a low 3DIRA had significantly shorter DFS than tumors with high 3D-IRA.



Fig. 3 A 75-year-old female patient. A squamous cell carcinoma (pT1bN0) is observed in the left lower lobe. a Lung window setting. b Mediastinal window setting at the early phase of contrast-enhanced CT. c lodine-enhanced image in contrast-enhanced CT. The 3D-IRA is 48 HU, and the corrected 3D-IRA is 6.45. The patient was recurrence-free for 51 months after a left lower lobectomy 



Fig. 4 The graph shows Kaplan-Meier curves for disease-free survival according to the corrected 3D-IRA at the early phase of contrast-enhanced CT. a Patients with tumors showing 3D-IRA $>5.88$ had a significantly better prognosis than those with tumors showing 3D-IRA $<5.88(p=0.017)$. b Patients with adenocarcinomas, $\mathbf{c}$ patients with squamous cell carcinomas, $\mathbf{d}$ patients with other subtype tumors showing $3 \mathrm{D}-\mathrm{IRA}>5.88 \mathrm{had}$ a better prognosis than those with tumors showing 3D-IRA $<5.88$ although there was no significant difference $(p=0.224, p=0.126$, and $p=0.109$, respectively)

The tumors with low 3D-IRA tended to show higher TNM stage because of pleural invasion, chest wall invasion, intrapulmonary metastasis, and lymph node metastases. The stage IB-III tumors showed significantly lower 3D-IRA at the early phase than the stage IA tumors. In addition, logistic regression analysis revealed that tumor size and corrected 3D-IRA at early phase were significant factors for stage IB or higher tumors. A few previous studies have shown that the 3D-IRA significantly correlates with the locoregional invasiveness of lung 
cancers, which is consistent with the current results [15, 16]. It is presumed that the strong invasiveness of the tumor will lead to postoperative recurrence even after complete resection. The recurrent tumors included a high rate of stage IB or higher tumors, and these showed significantly lower 3D-IRA than the non-recurrent tumors.

The ROC analysis indicated that the suitable cutoff value of corrected 3D-IRA at early phase was 5.88 for recurrence. This value represents $37 \mathrm{HU}$ for a patient weighing $50 \mathrm{~kg}$ with a contrast media dose of 320 $\mathrm{mgI}$ and $36 \mathrm{HU}$ for a patient weighing $60 \mathrm{~kg}$ with a contrast media dose of $370 \mathrm{mgI}$. In previous literature examining 3D-IRA and tumor differentiation, these values correspond to Grade 2 and Grade 3 boundary values [12]. Therefore, tumors with corrected 3DIRA $<5.88$ might include many poorly or undifferentiated carcinomas. When this cutoff value was used to divide the patients into two groups, 3D-IRA $<5.88$ showed significantly poorer prognosis on the KaplanMeier survival curve. These curves indicate that 3DIRA has a significant impact on tumor recurrence after 1 year post-surgery. We examined Kaplan-Meier survival curves for each pathological subtype, and for all subtypes, 3D-IRA $<5.88$ tumors showed a poor prognosis.

In recent years, stereotactic radiotherapy (SRT) has been increasingly used as an alternative to surgery for small-sized lung cancer. The weakness of SRT is that the histopathological invasiveness of the tumor cannot be determined surgically. For small-sized lung tumors, it is sometimes difficult to determine the histopathology using transbronchial lung biopsy and CT-guided transcutaneous needle biopsy. Therefore, it is more important to predict the invasiveness and prognosis of the tumor by radiological imaging before treatment. One previous study reported that the IRA at the early phase of contrast DE-CT is useful in predicting recurrence after SRT [19]. Although surgery and SRT are different treatment modalities, our results are consistent with their conclusions. Therefore, it is presumed that IRA at the early phase reflects the invasiveness and grade of the tumor rather than the responsiveness of the tumor to radiation therapy.

This study has several limitations. First, it was a retrospective and a single-center study. Second, we were not able to examine postoperative adjuvant therapy in detail in patients transferred to the hospital. However, adjuvant chemotherapy might not substantially affect our results because it was performed significantly more cases in the recurrence group. Finally, in this retrospective study, we were not able to examine the relationship between 3DIRA and pathological genetic prognostic markers such as Ki-67 and EGFR mutation [21-23].

\section{Conclusions}

The 3D-IRA of small-sized solid lung cancers measured by contrast-enhanced DE-CT was significantly associated with pathological invasiveness and postoperative recurrence. Tumors with a low 3D-IRA at the early phase tended to have a higher TNM stage and a significantly poorer prognosis.

\section{Abbreviations \\ 3D-IRA: Three-dimensional iodine-related attenuation; AUC: Area under the curve; cStage: clinical TNM staging; DE-CT: Dual-energy computed \\ tomography; EBUS-TBNA: Endobronchial ultrasound-guided trans-bronchial needle aspiration; pSize: pathological tumor size; pStage: pathological TNM staging; ROI: Region of interest; ROC: Receiver operating characteristic; SqCC: Squamous cell carcinoma; WWR: Wide wedge resection}

\section{Acknowledgements}

The authors thank Editage (www.editage.jp) for English language editing.

\section{Authors' contributions}

SI conceived of the present idea. SiN supervised the project. SI, SK, RI and ShN acquired, analyzed and interpreted the patient data. SI was major contributors in writing the manuscript. SK, RI, ShN, and SiN contributed to the revision of the manuscript. All authors have read and approved the final article.

\section{Funding}

This work was supported by KAKENHI (19 K08149) from the Japanese Ministry of Education, Culture, Sports, Science and Technology (MEXT).

\section{Availability of data and materials}

Not applicable.

\section{Ethics approval and consent to participate}

This retrospective study was approved by our institutional review board with a waiver of informed consent for retrospective data analysis of patients with surgically and histologically confirmed lung cancer who underwent DE-CT for a clinical indication at our institution (No. 2018-0434).

Consent for publication

Not applicable.

\section{Competing interests}

The authors declare that they have no competing interests.

\section{Author details}

${ }^{1}$ Department of Radiology, Nagoya University Graduate School of Medicine, 65 Tsurumai-cho, Showa-ku, Nagoya 466-8550, Japan. ${ }^{2}$ Department of Thoracic Surgery, Nagoya University Graduate School of Medicine, 65 Tsurumai-cho, Showa-ku, Nagoya 466-8550, Japan.

Received: 3 June 2020 Accepted: 11 December 2020

Published online: 07 January 2021

\section{References}

1. Rami-Porta R, Bolejack V, Crowley J, et al. The IASLC lung Cancer staging project: proposals for the revisions of the $T$ descriptors in the forthcoming eighth edition of the TNM classification for lung Cancer. J Thorac Oncol. 2015;10(7):990-1003. https://doi.org/10.1097/JTO.0000000000000559.

2. Travis WD, Asamura H, Bankier AA, et al. The IASLC lung Cancer staging project: proposals for coding $T$ categories for subsolid nodules and assessment of tumor size in part-solid tumors in the forthcoming eighth edition of the TNM classification of lung Cancer. J Thorac Oncol. 2016;11(8): 1204-23. https://doi.org/10.1016/j.jtho.2016.03.025.

3. Kamiya S, Iwano S, Umakoshi H, et al. Computer-aided Volumetry of partsolid lung cancers by using CT: solid component size predicts prognosis. Radiology. 2018;287(3):1030-40. https://doi.org/10.1148/radiol.2018172319.

4. Kishimoto M, Iwano S, Ito S, Kato K, Ito R, Naganawa S. Prognostic evaluations of small size lung cancers by $18 \mathrm{~F}-\mathrm{FDG}$ PET/CT and thin- 
section CT. Lung Cancer. 2014;86(2):180-4. https://doi.org/10.1016/j. lungcan.2014.09.006.

5. Furumoto H, Shimada Y, Imai K, et al. Prognostic impact of the integration of volumetric quantification of the solid part of the tumor on 3DCT and FDG-PET imaging in clinical stage IA adenocarcinoma of the lung. Lung Cancer. 2018;121:91-6. https://doi.org/10.1016/j.lungcan.2018.05.001.

6. Yamanashi K, Okumura N, Yamamoto Y, Takahashi A, Nakashima T, Matsuoka T. Comparing part-solid and pure-solid tumors in the TNM classification of lung Cancer (eighth edition). Thorac Cardiovasc Surg. 2019, 67(4):306-14. https://doi.org/10.1055/s-0038-1651521.

7. Fu F, Zhang Y, Wen Z, et al. Distinct prognostic factors in patients with stage I non-small cell lung Cancer with radiologic part-solid or solid lesions. J Thorac Oncol. 2019;14(12):2133-42. https://doi.org/10.1016/j.tho.2019.08.002.

8. Jiang T, Li M, Lin M, Zhao M, Zhan C, Feng M. Meta-analysis of comparing part-solid and pure-solid tumors in patients with clinical stage IA non-smallcell lung cancer in the eighth edition TNM classification. Cancer Manag Res. 2019;11:2951-61. https://doi.org/10.2147/CMAR.S196613.

9. Iwano S, Umakoshi H, Kamiya S, et al. Postoperative recurrence of clinical early-stage non-small cell lung cancers: a comparison between solid and subsolid nodules. Cancer Imaging. 2019;19(1):33. https://doi.org/10.1186/ s40644-019-0219-3.

10. Kinoshita F, Toyokawa G, Matsubara T, et al. Prognosis of early-stage partsolid and pure-solid lung adenocarcinomas. Anticancer Res. 2019;39(5): 2665-70. http://ar.iiarjournals.org/content/39/5/2665.

11. Schmid-Bindert G, Henzler T, Chu TQ, et al. Functional imaging of lung cancer using dual energy $C T$ : how does iodine related attenuation correlate with standardized uptake value of 18FDG-PET-CT? Eur Radiol. 2012;22(1):93103. https://doi.org/10.21873/anticanres.13391

12. Iwano $S$, Ito R, Umakoshi H, Ito S, Naganawa S. Evaluation of lung cancer by enhanced dual-energy CT: association between three-dimensional iodine concentration and tumour differentiation. Br J Radiol. 2015;88(1055): 20150224. https://doi.org/10.1259/bjr.20150224.

13. Gonzalez-Perez V, Arana E, Barrios M, et al. Differentiation of benign and malignant lung lesions: dual-energy computed tomography findings. Eur J Radiol. 2016;85(10):1765-72. https://doi.org/10.1016/j.jrad.2016.07.019.

14. Kim H, Park CM, Kang CK, Yoon J, Chae KJ, Goo JM. Effect of CT acquisition parameters on iodine density measurement at dual-layer spectral CT. AJR Am J Roentgenol. 2018;211(4):748-54. https://doi.org/ 10.1016/j.ejrad.2016.07.019.

15. Ito $\mathrm{R}$, Iwano $\mathrm{S}$, Shimamoto $\mathrm{H}$, et al. A comparative analysis of dual-phase dual-energy $C T$ and FDG-PET/CT for the prediction of histopathological invasiveness of non-small cell lung cancer. Eur J Radiol. 2017;95:186-91. https://doi.org/10.1016/j.ejrad.2017.08.010.

16. Shimamoto H, Iwano S, Umakoshi H, Kawaguchi K, Naganawa S. Evaluation of locoregional invasiveness of small-sized non-small cell lung cancers by enhanced dual-energy computed tomography. Cancer Imaging. 2016;16(1): 18. https://doi.org/10.1186/s40644-016-0077-1.

17. Fehrenbach U, Kahn J, Boning G, et al. Spectral $C T$ and its specific values in the staging of patients with non-small cell lung cancer: technical possibilities and clinical impact. Clin Radiol. 2019;74(6):456-66. https://doi. org/10.1016/j.crad.2019.02.010

18. Lin LY, Zhang Y, Suo ST, Zhang F, Cheng JJ, Wu HW. Correlation between dual-energy spectral $C T$ imaging parameters and pathological grades of non-small cell lung cancer. Clin Radiol. 2018;73(4):412 e411-7. https://doi. org/10.1016/j.crad.2017.11.004.

19. Aoki M, Hirose K, Sato M, et al. Prognostic impact of average iodine density assessed by dual-energy spectral imaging for predicting lung tumor recurrence after stereotactic body radiotherapy. J Radiat Res. 2016;57(4):3816. https://doi.org/10.1093/jrr/rrv100.

20. Fehrenbach U, Feldhaus F, Kahn J, et al. Tumour response in non-small-cell lung cancer patients treated with chemoradiotherapy - can spectral CT predict recurrence? J Med Imaging Radiat Oncol. 2019;63(5):641-9. https:// doi.org/10.1111/1754-9485.12926.

21. Chen M, Li X, Wei Y, Qi L, Sun YS. Spectral CT imaging parameters and Ki-67 labeling index in lung adenocarcinoma. Chin J Cancer Res. 2020;32(1):96104. https://doi.org/10.21147/j.issn.1000-9604.2020.01.11.

22. Li M, Zhang L, Tang W, et al. Dual-energy spectral CT characteristics in surgically resected lung adenocarcinoma: comparison between Kirsten rat sarcoma viral oncogene mutations and epidermal growth factor receptor mutations. Cancer Imaging. 2019;19(1):77. https://doi.org/10.1186/s40644019-0261-1.
23. Han X, Fan J, Gu J, Li Y, Yang M, Liu T, Li N, Zeng W, Shi H. CT features associated with EGFR mutations and ALK positivity in patients with multiple primary lung adenocarcinomas. Cancer Imaging. 2020;20(1):51. https://doi. org/10.1186/s40644-020-00330-1.

\section{Publisher's Note}

Springer Nature remains neutral with regard to jurisdictional claims in published maps and institutional affiliations.
Ready to submit your research? Choose BMC and benefit from:

- fast, convenient online submission

- thorough peer review by experienced researchers in your field

- rapid publication on acceptance

- support for research data, including large and complex data types

- gold Open Access which fosters wider collaboration and increased citations

- maximum visibility for your research: over $100 \mathrm{M}$ website views per year

At BMC, research is always in progress.

Learn more biomedcentral.com/submissions 